\title{
A Study on the Refresher Training of Physical Therapist in Gwangju and Jeonnam
}

\author{
Seong-Hun Yu', Seung-Rae Kim², Sung-Hyoun Cho ${ }^{3}$, Il-Yong Jang ${ }^{4}$, Jin-Ah Hwang ${ }^{5}$, Yong-Seong Kim³, Hyun-Jin Kim ${ }^{6}$ \\ ${ }^{1}$ Gwangju Trauma Center, Gwangju; ${ }^{2}$ Gwangju Donggu Public Health Center, Gwangju; ${ }^{3}$ Department of Physical Therapy, Nambu University, \\ Gwangju; ${ }^{4}$ Suwan Medical Center, Gwangju; ${ }^{5}$ X-sports Rehabilitation Exercise Center, Gwangju; ${ }^{6}$ Department of Physical Therapy, Hanlyo University, \\ Gwangju, Korea
}

Purpose: The current study seeks to examine the continuing education of physical therapist in Gwangju, Jeonnam.

Methods: A survey was conducted during the continuing education of physical therapist held in Gwangju in 2015 with 297 participants. The survey questionnaire consisted of 13 questions on characteristics of physical therapist, 7 questions on the level of satisfaction with continuing education, 11 questions regarding the need for continuing education, and 8 questions concerning how to improve continuing education.

Results: As for the level of satisfaction with the content of continuing education, the survey results indicated that there were significant differences across respondents' age and career period. Regarding the level of satisfaction with the environment in which continuing education was provided; significant differences were observed across respondents' age, education, marital status, monthly pay, career period, and service period. Regarding the level of satisfaction with the expense in which continuing education was provided; significant differences were observed across respondents' sex, age, education, marital status, dependent family, monthly pay, career period, and position. In terms of the level of satisfaction with the operation method in which continuing education was carried out, there were significant differences across respondents' age, education, and career period.

Conclusion: The survey found the level of satisfaction with continuing education to be average among physical therapist in Gwangju and Jeonnam. In addition, as for ways to take continuing education courses for those who have never taken it, online education was mentioned the most. Therefore, there is a need for advertising online continuing education.

Keywords: Continuing education, Physical therapist, Satisfaction

\section{INTRODUCTION}

With changes in healthcare environment and an increase in national income and public awareness of right to know, people's demand for quality medical services is rising ever more. ${ }^{1}$ In addition, since medical skills and knowledge are evolving at unprecedented speeds, there should be a persistent effort to organically embrace the skills and knowledge of medical professionals and develop their expertise. ${ }^{2,3}$

Therefore, in order to meet the needs of the changing healthcare environment and people, appropriate education is needed which is most critical to building the capacity of individuals and to surviving

Received May 20, 2016 Revised Jun 14, 2016

Accepted Jun 29, 2016

Corresponding author Hyun-Jin Kim

E-mail gini51@naver.com in the era of infinite competition.-6 Physical therapists should receive eight hours or more of continuing education every year in pursuance of Article 20 of the Act on medical professionals.? The education is mandatory for every physical therapist and is part of their lifelong education to acquire up-to-date knowledge and information while complementing and supplementing the skills and knowledge needed for the profession. ${ }^{8}$ Such continuing education must be in line with trainees' current work and should be aimed at improving their capacity to carry out the roles and responsibilities involved. ${ }^{9,10}$

However, in the past, many physical therapists could not participate in the education due to various limitations even though con-

Copylight (C2016 The Korea Society of Physical Therapy

This is an Open Access article distribute under the terms of the Creative Commons Attribution Non-commercial License (Http:// creativecommons.org/license/by-nc/4.o.) which permits unrestricted non-commercial use, distribution, and reproduction in any medium, provided the original work is properly cited. 
tinuing education was essential for them. Nevertheless, with the recent medical license reporting system, continuing education has become more stringent and binding. ${ }^{11}$ Although continuing education has now been made highly mandatory, the awareness of and needs for such education among physical therapists is modest at best. Therefore, it is very important to conduct research into the continuing education for physical therapists, and to analyze and evaluate the current continuing education to see whether it has achieved its original purpose.

The needs to license update existing health care provider and medical technician have been raised due to various medical emergencies. Because of these reasons were studied to increase the continuing education quality. Hence, the present study aims to provide baseline data for the quality improvement of continuing education for physical therapists by examining the current status of education and investigating the awareness of and needs for continuing education among physical therapists.

\section{METHODS}

\section{Subjects}

A survey was conducted at one of the continuing education programs for physical therapists in Gwangju in 2015 with the therapists who had agreed to take part in the survey after being informed of the survey purpose. A total of 297 respondents participated in the survey.

\section{Experimental methods}

The survey questionnaire consisted of 13 questions about the general characteristics of physical therapists, 7 questions about satisfaction with continuing education, 11 questions about the needs for continuing education, and 8 questions about improvement of continuing education. This survey was made on the basis of the literature; physical therapy processor and doctor's degree decided appropriate question number and details. ${ }^{12-15}$ The survey was filled out anonymously. With regard to the questions about satisfaction with continuing education in particular, the respondents were asked to rate their level of satisfaction on a five-point scale with one being very dissatisfied, two being dissatisfied, three being neutral, four being satisfied and five being very satisfied, and moreover, on the questions about improvement of continuing education, the respon- dents could give as many as three answers.

\section{Data analysis}

The survey responses were analyzed using SPSS 18.0 software. For the data of the respondents' general characteristics and questions about continuing education, frequency analysis was used while an independent t-test was conducted to investigate the respondents' satisfaction with continuing education by their general characteristics such as gender, marital status, number of dependents, job position and place of residence. In addition, an analysis of variance (ANOVA) was conducted to examine the satisfaction with continuing education by group with regard to the age, educational level, remuneration, work hours, employment history and job responsibilities of the respondents and the number of patients they oversee. A post hoc test was carried out using Tukey's method. The statistical significance was set at $\alpha=0.05$.

\section{RESULTS}

\section{General characteristics of physical therapist}

There were a total of 297 physical therapists participating in the survey, of whom $67.3 \%$ were female and $42.4 \%$ were the respondents aged between 30-39 years which constituted the largest group. Most of the respondents were college graduates 59.6\%, unmarried $61.6 \%$ and did not have any dependent family members 57.6\%. In addition, a majority of them were paid between 1,510,000-2,000,000 won per month on average $43.8 \%$, working for five years or less $38.0 \%$ and held the position of a physical therapist $72.1 \%$. Furthermore, a lion's share of them had the working week of between $41-49$ hours $70.0 \%$, were living in Gwangju 76.1\%, had the service period of five years or less $73.4 \%$, were in part of electrotherapy physical therapy $45.1 \%$ and had 31-40 patients per day 24.9\% (Table 1).

\section{Level of satisfaction with continuing education in physical}

\section{therapist}

With regard to the level of satisfaction with continuing education among physical therapists, $61.6 \%$ of the respondents were satisfied with the education content while the satisfaction levels for the method, environment, instructors, hours and operation method of continuing education were $62.3 \%, 52.5 \%, 68.0 \%, 56.6 \%$, and $66.3 \%$, respectively. A majority of their responses were neutral. However, 
Table 1. General characteristics of physical therapist

\begin{tabular}{|c|c|c|c|}
\hline Characteristics & & $\mathrm{N}$ & $\%$ \\
\hline Sex & $\begin{array}{l}\text { Male } \\
\text { Female }\end{array}$ & $\begin{array}{r}97 \\
200\end{array}$ & $\begin{array}{l}32.7 \\
67.3\end{array}$ \\
\hline Age & $\begin{array}{l}\text { 20-29 years old } \\
30-39 \text { years old } \\
<40 \text { years old }\end{array}$ & $\begin{array}{r}116 \\
126 \\
55\end{array}$ & $\begin{array}{l}39.1 \\
42.4 \\
18.5\end{array}$ \\
\hline Education & $\begin{array}{l}\text { College graduate } \\
\text { University graduate } \\
\text { A postgraduate school }\end{array}$ & $\begin{array}{r}177 \\
97 \\
23\end{array}$ & $\begin{array}{r}59.6 \\
32.7 \\
7.7\end{array}$ \\
\hline Marital status & $\begin{array}{l}\text { Unmarried } \\
\text { Married }\end{array}$ & $\begin{array}{l}183 \\
114\end{array}$ & $\begin{array}{l}61.6 \\
38.4\end{array}$ \\
\hline Dependent family & $\begin{array}{l}\text { No } \\
\text { Yes }\end{array}$ & $\begin{array}{l}171 \\
126\end{array}$ & $\begin{array}{l}57.6 \\
42.4\end{array}$ \\
\hline Monthly pay & $\begin{array}{l}<1,500,000 \\
1,510,000-2,000,000 \\
2,010,000-2,500,000 \\
2,510,000-3,000,000 \\
<3,010,000\end{array}$ & $\begin{array}{r}48 \\
130 \\
57 \\
42 \\
20\end{array}$ & $\begin{array}{r}16.2 \\
43.8 \\
19.2 \\
14.1 \\
6.7\end{array}$ \\
\hline $\begin{array}{l}\text { Physical therapist } \\
\text { career period }\end{array}$ & $\begin{array}{l}<5 \text { years old } \\
6-9 \text { years old } \\
<10 \text { years old }\end{array}$ & $\begin{array}{r}113 \\
106 \\
78\end{array}$ & $\begin{array}{l}38.0 \\
35.7 \\
26.3\end{array}$ \\
\hline Position & $\begin{array}{l}\text { Manager of physical therapy } \\
\text { Physical therapy } \\
\text { And so on }\end{array}$ & $\begin{array}{r}76 \\
214 \\
7\end{array}$ & $\begin{array}{r}25.6 \\
72.1 \\
2.4\end{array}$ \\
\hline Working week & $\begin{array}{l}<40 \text { times } \\
41-49 \text { times } \\
<50 \text { times }\end{array}$ & $\begin{array}{r}66 \\
208 \\
23\end{array}$ & $\begin{array}{r}22.2 \\
70.0 \\
7.7\end{array}$ \\
\hline Site & $\begin{array}{l}\text { Gwangju } \\
\text { Jeonnam }\end{array}$ & $\begin{array}{r}226 \\
71\end{array}$ & $\begin{array}{l}76.1 \\
23.9\end{array}$ \\
\hline Service period & $\begin{array}{l}<5 \text { years old } \\
6-9 \text { years old } \\
<10 \text { years old }\end{array}$ & $\begin{array}{r}218 \\
58 \\
21\end{array}$ & $\begin{array}{r}73.4 \\
19.5 \\
7.1\end{array}$ \\
\hline Part & $\begin{array}{l}\text { Neurological physical therapy } \\
\text { Electrotherapy physical therapy } \\
\text { Pediatric physical therapy } \\
\text { Orthopedic physical therapy }\end{array}$ & $\begin{array}{r}65 \\
134 \\
43 \\
55\end{array}$ & $\begin{array}{l}21.9 \\
45.1 \\
14.5 \\
18.5\end{array}$ \\
\hline $\begin{array}{l}1 \text { day number of } \\
\text { patient care }\end{array}$ & $\begin{array}{l}<10 \text { peoples } \\
11-20 \text { peoples } \\
21-30 \text { peoples } \\
31-40 \text { peoples } \\
<41 \text { peoples } \\
\text { And so on }\end{array}$ & $\begin{array}{l}25 \\
67 \\
71 \\
74 \\
47 \\
13\end{array}$ & $\begin{array}{r}8.4 \\
22.6 \\
23.9 \\
24.9 \\
15.8 \\
4.4\end{array}$ \\
\hline
\end{tabular}

with regard to the education expense, the largest number of them 38.7\% were dissatisfied (Table 2).

\section{Level of satisfaction with continuing education in terms of physical therapist characteristics}

The satisfaction level for the content of continuing education was significantly different by age and physical therapist career period while there were significant differences in the satisfaction level for the environment of continuing education by age, educational level, marital status, monthly pay, physical therapist career period and service period. In addition, with regard to the satisfaction level for the expense of continuing education, there were significant differ-
Table 2. Level of satisfaction with continuing education in physical therapist

\begin{tabular}{|c|c|c|c|}
\hline Characteristics & & $\mathrm{N}$ & $\%$ \\
\hline $\begin{array}{l}\text { Are you satisfied with the content } \\
\text { of continuing education? }\end{array}$ & $\begin{array}{l}\text { Very dissatisfied } \\
\text { Dissatisfied } \\
\text { Neutral } \\
\text { Satisfied } \\
\text { Very satisfied }\end{array}$ & $\begin{array}{r}19 \\
55 \\
183 \\
38 \\
2\end{array}$ & $\begin{array}{r}6.4 \\
18.5 \\
61.6 \\
12.8 \\
0.7\end{array}$ \\
\hline $\begin{array}{l}\text { Are you satisfied with the method } \\
\text { of continuing education? }\end{array}$ & $\begin{array}{l}\text { Very dissatisfied } \\
\text { Dissatisfied } \\
\text { Neutral } \\
\text { Satisfied } \\
\text { Very satisfied }\end{array}$ & $\begin{array}{r}14 \\
56 \\
185 \\
37 \\
5\end{array}$ & $\begin{array}{r}4.7 \\
18.9 \\
62.3 \\
12.5 \\
1.7\end{array}$ \\
\hline $\begin{array}{l}\text { Are you satisfied with the } \\
\text { environment of continuing } \\
\text { education? }\end{array}$ & $\begin{array}{l}\text { Very dissatisfied } \\
\text { Dissatisfied } \\
\text { Neutral } \\
\text { Satisfied } \\
\text { Very satisfied }\end{array}$ & $\begin{array}{r}11 \\
45 \\
156 \\
79 \\
6\end{array}$ & $\begin{array}{r}3.7 \\
15.2 \\
52.5 \\
26.6 \\
2.0\end{array}$ \\
\hline $\begin{array}{l}\text { Are you satisfied with the instructor } \\
\text { of continuing education? }\end{array}$ & $\begin{array}{l}\text { Very dissatisfied } \\
\text { Dissatisfied } \\
\text { Neutral } \\
\text { Satisfied } \\
\text { Very satisfied }\end{array}$ & $\begin{array}{r}10 \\
32 \\
202 \\
49 \\
4\end{array}$ & $\begin{array}{r}3.4 \\
10.8 \\
68.0 \\
16.5 \\
1.3\end{array}$ \\
\hline $\begin{array}{l}\text { Are you satisfied with the hour } \\
\text { of continuing education? }\end{array}$ & $\begin{array}{l}\text { Very dissatisfied } \\
\text { Dissatisfied } \\
\text { Neutral } \\
\text { Satisfied } \\
\text { Very satisfied }\end{array}$ & $\begin{array}{r}18 \\
72 \\
168 \\
37 \\
2\end{array}$ & $\begin{array}{r}6.1 \\
24.2 \\
56.6 \\
12.5 \\
0.7\end{array}$ \\
\hline $\begin{array}{l}\text { Are you satisfied with the expense } \\
\text { of continuing education? }\end{array}$ & $\begin{array}{l}\text { Very dissatisfied } \\
\text { Dissatisfied } \\
\text { Neutral } \\
\text { Satisfied } \\
\text { Very satisfied }\end{array}$ & $\begin{array}{r}64 \\
115 \\
107 \\
11 \\
0\end{array}$ & $\begin{array}{r}21.5 \\
38.7 \\
36.0 \\
3.7 \\
0\end{array}$ \\
\hline $\begin{array}{l}\text { Are you satisfied with the operation } \\
\text { method of continuing education? }\end{array}$ & $\begin{array}{l}\text { Very dissatisfied } \\
\text { Dissatisfied } \\
\text { Neutral } \\
\text { Satisfied } \\
\text { Very satisfied }\end{array}$ & $\begin{array}{r}18 \\
54 \\
197 \\
26 \\
2\end{array}$ & $\begin{array}{r}6.1 \\
18.2 \\
66.3 \\
8.8 \\
0.7\end{array}$ \\
\hline
\end{tabular}

ences by sex, age, educational level, marital status, number of dependent family members, monthly pay, physical therapist career period, and job position. Moreover, the satisfaction level for the operation method of continuing education was significantly different according to age, educational level and physical therapist career period $(\mathrm{p}<0.05)$. However, no significant difference was observed in the satisfaction levels for the method, instructors, and hours of continuing education (Table 3).

\section{Need for continuing education}

On the question of "Do you satisfied with the contents of the continuing education?", $85.9 \%$ of the respondents answered they were "very dissatisfied". The question of "do you think continuing education is needed?", most of the respondents' answers $48.8 \%$ were "neutral". In addition, $40.4 \%$ of them answered "neutral", when asked "how helpful do you think continuing education is to your work?" 
Table 3. The level of satisfaction with continuing education in terms of physical therapist characteristics

\begin{tabular}{|c|c|c|c|c|c|}
\hline \multicolumn{3}{|l|}{ Characteristics } & \multirow{2}{*}{$\begin{array}{l}\mathrm{N} \\
97\end{array}$} & \multirow{2}{*}{$\frac{\mathrm{M} \pm \mathrm{SD}}{2.84 \pm 0.77}$} & \multirow{2}{*}{$\frac{\text { tor } F}{0.10}$} \\
\hline Are you satisfied with the content & Sex & Male & & & \\
\hline \multirow{39}{*}{ of continuing education? } & & Female & 200 & $2.83 \pm 0.75$ & \\
\hline & Age & 20-29 years old & 116 & $2.72 \pm 0.74$ & $2.85^{*}$ \\
\hline & & 30-39 years old & 126 & $2.86 \pm 0.80$ & \\
\hline & & <40 years old & 55 & $3.00 \pm 0.64$ & \\
\hline & Education & College graduate & 177 & $2.82 \pm 0.75$ & 1.62 \\
\hline & & University graduate & 97 & $2.77 \pm 0.78$ & \\
\hline & & A postgraduate school & 23 & $3.09 \pm 0.67$ & \\
\hline & Marital status & Unmarried & 183 & $2.79 \pm 0.06$ & -1.04 \\
\hline & & Married & 114 & $2.89 \pm 0.07$ & \\
\hline & Dependent family & No & 171 & $2.80 \pm 0.76$ & -0.87 \\
\hline & & Yes & 126 & $2.87 \pm 0.75$ & \\
\hline & Monthly pay & $<1,500,000$ & 48 & $2.77 \pm 0.95$ & 0.80 \\
\hline & & $1,510,000-2,000,000$ & 130 & $2.83 \pm 0.66$ & \\
\hline & & $2,010,000-2,500,000$ & 57 & $2.88 \pm 0.71$ & \\
\hline & & $2,510,000-3,000,000$ & 42 & $2.71 \pm 0.86$ & \\
\hline & & $<3,010,000$ & 20 & $3.05 \pm 0.69$ & \\
\hline & Physical therapist career period & $<5$ years old & 113 & $2.81 \pm 0.74$ & $3.78^{*}$ \\
\hline & & 6-9 years old & 106 & $2.71 \pm 0.84$ & \\
\hline & & $<10$ years old & 78 & $3.01 \pm 0.61$ & \\
\hline & Position & Manager of physical therapy & 76 & $2.72 \pm 0.76$ & -1.53 \\
\hline & & Physical therapy & 214 & $2.88 \pm 0.75$ & \\
\hline & Working week & $<40$ times & 66 & $2.89 \pm 0.64$ & 1.13 \\
\hline & & 41-49 times & 208 & $2.79 \pm 0.81$ & \\
\hline & & $<50$ times & 23 & $3.00 \pm 0.52$ & \\
\hline & Site & Gwangju & 226 & $2.85 \pm 0.75$ & 0.68 \\
\hline & & Jeonnam & 71 & $2.77 \pm 0.78$ & \\
\hline & Service period & $<5$ years old & 218 & $2.81 \pm 0.75$ & 0.95 \\
\hline & & 6-9 years old & 58 & $2.81 \pm 0.83$ & \\
\hline & & $<10$ years old & 21 & $3.05 \pm 0.50$ & \\
\hline & Part & Neurological physical therapy & 65 & $2.82 \pm 0.58$ & 1.82 \\
\hline & & Electrotherapy physical therapy & 134 & $2.92 \pm 0.74$ & \\
\hline & & Pediatric physical therapy & 43 & $2.77 \pm 0.90$ & \\
\hline & & Orthopedic physical therapy & 55 & $2.65 \pm 0.82$ & \\
\hline & 1 day number of patient care & $<10$ peoples & 25 & $2.76 \pm 1.01$ & 0.91 \\
\hline & & 11-20 peoples & 67 & $2.90 \pm 0.63$ & \\
\hline & & 21-30 peoples & 71 & $2.94 \pm 0.70$ & \\
\hline & & 31-40 peoples & 74 & $2.70 \pm 0.79$ & \\
\hline & & <41 peoples & 47 & $2.81 \pm 0.83$ & \\
\hline & & And so on & 13 & $2.77 \pm 0.60$ & \\
\hline \multirow{22}{*}{$\begin{array}{l}\text { Are you satisfied with the method } \\
\text { of continuing education? }\end{array}$} & Sex & Male & 97 & $2.89 \pm 0.72$ & \\
\hline & & Female & 200 & $2.87 \pm 0.76$ & 0.18 \\
\hline & Age & 20-29 years old & 116 & $2.78 \pm 0.76$ & 1.74 \\
\hline & & 30-39 years old & 126 & $2.90 \pm 0.75$ & \\
\hline & & $<40$ years old & 55 & $3.00 \pm 0.69$ & \\
\hline & Education & College graduate & 177 & $2.85 \pm 0.74$ & 1.05 \\
\hline & & University graduate & 97 & $2.88 \pm 0.78$ & \\
\hline & & A postgraduate school & 23 & $3.09 \pm 0.60$ & \\
\hline & Marital status & Unmarried & 183 & $2.86 \pm 0.06$ & -0.35 \\
\hline & & Married & 114 & $2.89 \pm 0.07$ & \\
\hline & Dependent family & No & 171 & $2.88 \pm 0.75$ & 0.05 \\
\hline & & Yes & 126 & $2.87 \pm 0.74$ & \\
\hline & Monthly pay & $<1,500,000$ & 48 & $2.83 \pm 0.96$ & 0.64 \\
\hline & & $1,510,000-2,000,000$ & 130 & $2.85 \pm 0.65$ & \\
\hline & & $2,010,000-2,500,000$ & 57 & $2.96 \pm 0.76$ & \\
\hline & & $2,510,000-3,000,000$ & 42 & $2.81 \pm 0.77$ & \\
\hline & & $<3,010,000$ & 20 & $3.05 \pm 0.61$ & \\
\hline & Physical therapist career period & $<5$ years old & 113 & $2.88 \pm 0.73$ & 0.66 \\
\hline & & $6-9$ years old & 106 & $2.82 \pm 0.84$ & \\
\hline & & $<10$ years old & 78 & $2.95 \pm 0.62$ & \\
\hline & Position & Manager of physical therapy & 76 & $2.75 \pm 0.70$ & -1.55 \\
\hline & & Physical therapy & 214 & $2.93 \pm 0.75$ & \\
\hline
\end{tabular}


Table 3. Continued

\begin{tabular}{|c|c|c|c|c|c|}
\hline \multirow[t]{2}{*}{ Characteristics } & & & $\mathrm{N}$ & $\mathrm{M} \pm \mathrm{SD}$ & tor $F$ \\
\hline & Working week & $<40$ times & 66 & $2.95 \pm 0.64$ & \multirow[t]{3}{*}{1.74} \\
\hline & & 41-49 times & 208 & $2.83 \pm 0.77$ & \\
\hline & & $<50$ times & 23 & $3.09 \pm 0.73$ & \\
\hline & \multirow[t]{2}{*}{ Site } & Gwangju & 226 & $2.88 \pm 0.74$ & \multirow[t]{2}{*}{0.21} \\
\hline & & Jeonnam & 71 & $2.86 \pm 0.78$ & \\
\hline & \multirow{3}{*}{ Service period } & $<5$ years old & 218 & $2.88 \pm 0.74$ & \multirow[t]{3}{*}{0.21} \\
\hline & & $6-9$ years old & 58 & $2.86 \pm 0.83$ & \\
\hline & & $<10$ years old & 21 & $2.86 \pm 0.57$ & \\
\hline & \multirow[t]{4}{*}{ Part } & Neurological physical therapy & 65 & $2.83 \pm 0.68$ & \multirow[t]{4}{*}{0.06} \\
\hline & & Electrotherapy physical therapy & 134 & $3.00 \pm 0.73$ & \\
\hline & & Pediatric physical therapy & 43 & $2.72 \pm 0.83$ & \\
\hline & & Orthopedic physical therapy & 55 & $2.75 \pm 0.78$ & \\
\hline & \multirow[t]{6}{*}{1 day number of patient care } & $<10$ peoples & 25 & $3.00 \pm 1.04$ & \multirow[t]{6}{*}{1.09} \\
\hline & & 11-20 peoples & 67 & $2.79 \pm 0.66$ & \\
\hline & & 21-30 peoples & 71 & $3.01 \pm 0.49$ & \\
\hline & & 31-40 peoples & 74 & $2.78 \pm 0.82$ & \\
\hline & & <41 peoples & 47 & $2.89 \pm 0.89$ & \\
\hline & & And so on & 13 & $2.77 \pm 0.60$ & \\
\hline \multirow{40}{*}{$\begin{array}{l}\text { Are you satisfied with the environment } \\
\text { of continuing education? }\end{array}$} & \multirow[t]{2}{*}{ Sex } & Male & 97 & $3.12 \pm 0.77$ & \multirow[t]{2}{*}{0.64} \\
\hline & & Female & 200 & $3.06 \pm 0.82$ & \\
\hline & Age & 20-29 years old & 116 & $2.87 \pm 0.75$ & $9.11^{*}$ \\
\hline & & 30-39 years old & 126 & $3.13 \pm 0.81$ & \\
\hline & & $<40$ years old & 55 & $3.40 \pm 0.76$ & \\
\hline & Education & College graduate & 177 & $3.03 \pm 0.79$ & $2.66^{*}$ \\
\hline & & University graduate & 97 & $3.09 \pm 0.83$ & \\
\hline & & A postgraduate school & 23 & $3.43 \pm 0.73$ & \\
\hline & Marital status & Unmarried & 183 & $2.98 \pm 0.06$ & $-2.67^{*}$ \\
\hline & & Married & 114 & $3.24 \pm 0.08$ & \\
\hline & Dependent family & No & 171 & $3.02 \pm 0.79$ & -1.58 \\
\hline & & Yes & 126 & $3.17 \pm 0.82$ & \\
\hline & Monthly pay & $<1,500,000$ & 48 & $2.90 \pm 0.97$ & $2.46^{*}$ \\
\hline & & $1,510,000-2,000,000$ & 130 & $3.03 \pm 0.73$ & \\
\hline & & $2,010,000-2,500,000$ & 57 & $3.19 \pm 0.77$ & \\
\hline & & $2,510,000-3,000,000$ & 42 & $3.10 \pm 0.85$ & \\
\hline & & $<3,010,000$ & 20 & $3.50 \pm 0.69$ & \\
\hline & Physical therapist career period & $<5$ years old & 113 & $2.92 \pm 0.77$ & $6.85^{\star}$ \\
\hline & & $6-9$ years old & 106 & $3.06 \pm 0.82$ & \\
\hline & & $<10$ years old & 78 & $3.35 \pm 0.77$ & \\
\hline & Position & Manager of physical therapy & 76 & $3.11 \pm 0.78$ & 0.38 \\
\hline & & Physical therapy & 214 & $3.07 \pm 0.80$ & \\
\hline & Working week & $<40$ times & 66 & $3.11 \pm 0.75$ & 0.31 \\
\hline & & 41-49 times & 208 & $3.09 \pm 0.83$ & \\
\hline & & $<50$ times & 23 & $2.96 \pm 0.71$ & \\
\hline & Site & Gwangju & 226 & $3.09 \pm 0.81$ & 0.29 \\
\hline & & Jeonnam & 71 & $3.06 \pm 0.79$ & \\
\hline & Service period & $<5$ years old & 218 & $3.01 \pm 0.80$ & $4.73^{*}$ \\
\hline & & $6-9$ years old & 58 & $3.19 \pm 0.76$ & \\
\hline & & $<10$ years old & 21 & $3.52 \pm 0.75$ & \\
\hline & Part & Neurological physical therapy & 65 & $2.95 \pm 0.72$ & 0.23 \\
\hline & & Electrotherapy physical therapy & 134 & $3.17 \pm 0.78$ & \\
\hline & & Pediatric physical therapy & 43 & $3.12 \pm 1.01$ & \\
\hline & & Orthopedic physical therapy & 55 & $2.98 \pm 0.76$ & \\
\hline & 1 day number of patient care & $<10$ peoples & 25 & $3.04 \pm 1.02$ & 0.19 \\
\hline & & 11-20 peoples & 67 & $3.04 \pm 0.79$ & \\
\hline & & 21-30 peoples & 71 & $3.13 \pm 0.68$ & \\
\hline & & 31-40 peoples & 74 & $3.05 \pm 0.86$ & \\
\hline & & $<41$ peoples & 47 & $3.15 \pm 0.78$ & \\
\hline & & And so on & 13 & $3.00 \pm 0.91$ & \\
\hline Are you satisfied with the instructor & Sex & Male & 97 & $3.00 \pm 0.77$ & -0.29 \\
\hline of continuing education? & & Female & 200 & $3.03 \pm 0.64$ & \\
\hline & Age & 20-29 years old & 116 & $3.00 \pm 0.59$ & 0.39 \\
\hline & & 30-39 years old & 126 & $3.00 \pm 0.75$ & \\
\hline & & <40 years old & 55 & $3.09 \pm 0.70$ & \\
\hline
\end{tabular}


Table 3. Continued

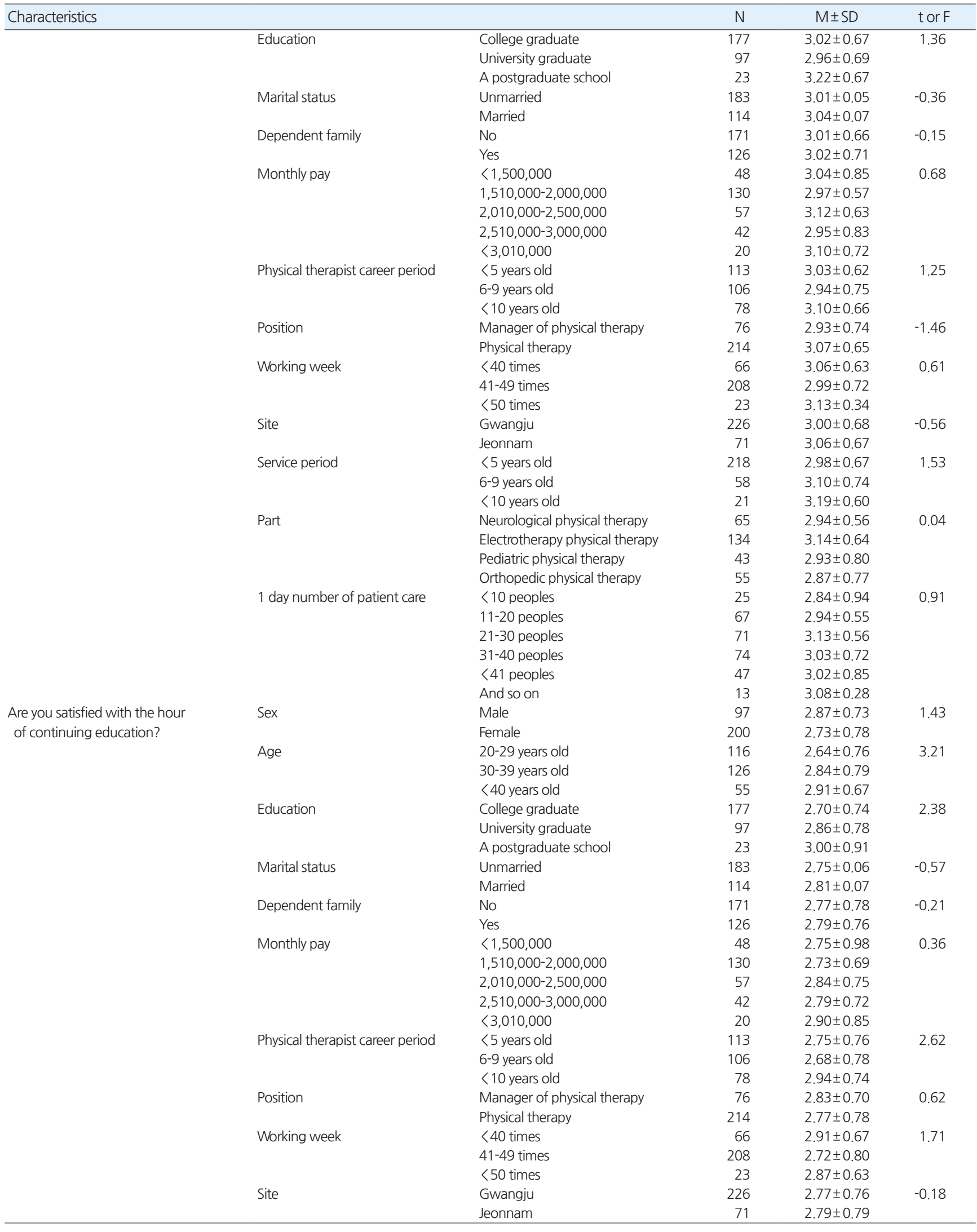


Table 3. Continued

\begin{tabular}{|c|c|c|c|c|c|}
\hline \multirow[t]{2}{*}{ Characteristics } & & & $\mathrm{N}$ & $\mathrm{M} \pm \mathrm{SD}$ & tor $F$ \\
\hline & \multirow[t]{3}{*}{ Service period } & $<5$ years old & 218 & $2.77 \pm 0.76$ & 0.14 \\
\hline & & 6-9 years old & 58 & $2.76 \pm 0.78$ & \\
\hline & & $<10$ years old & 21 & $2.86 \pm 0.79$ & \\
\hline & \multirow[t]{4}{*}{ Part } & Neurological physical therapy & 65 & $2.72 \pm 0.60$ & 0.59 \\
\hline & & Electrotherapy physical therapy & 134 & $2.84 \pm 0.77$ & \\
\hline & & Pediatric physical therapy & 43 & $2.67 \pm 0.94$ & \\
\hline & & Orthopedic physical therapy & 55 & $2.76 \pm 0.79$ & \\
\hline & \multirow[t]{6}{*}{1 day number of patient care } & $<10$ peoples & 25 & $2.80 \pm 0.91$ & 0.55 \\
\hline & & 11-20 peoples & 67 & $2.73 \pm 0.61$ & \\
\hline & & 21-30 peoples & 71 & $2.86 \pm 0.70$ & \\
\hline & & 31-40 peoples & 74 & $2.70 \pm 0.84$ & \\
\hline & & <41 peoples & 47 & $2.85 \pm 0.83$ & \\
\hline & & And so on & 13 & $2.62 \pm 0.87$ & \\
\hline \multirow{40}{*}{$\begin{array}{l}\text { Are you satisfied with the expense } \\
\text { of continuing education? }\end{array}$} & \multirow[t]{2}{*}{ Sex } & Male & 97 & $2.47 \pm 0.86$ & $3.68^{*}$ \\
\hline & & Female & 200 & $2.10 \pm 0.78$ & \\
\hline & \multirow[t]{3}{*}{ Age } & 20-29 years old & 116 & $1.98 \pm 0.75$ & $10.65^{\star}$ \\
\hline & & 30-39 years old & 126 & $2.29 \pm 0.86$ & \\
\hline & & $<40$ years old & 55 & $2.56 \pm 0.76$ & \\
\hline & \multirow[t]{3}{*}{ Education } & College graduate & 177 & $2.10 \pm 0.80$ & $7.25^{*}$ \\
\hline & & University graduate & 97 & $2.31 \pm 0.83$ & \\
\hline & & A postgraduate school & 23 & $2.74 \pm 0.75$ & \\
\hline & \multirow[t]{2}{*}{ Marital status } & Unmarried & 183 & $2.10 \pm 0.06$ & $-3.24^{*}$ \\
\hline & & Married & 114 & $2.41 \pm 0.08$ & \\
\hline & \multirow[t]{2}{*}{ Dependent family } & No & 171 & $2.12 \pm 0.83$ & $-2.36^{*}$ \\
\hline & & Yes & 126 & $2.35 \pm 0.80$ & \\
\hline & \multirow[t]{5}{*}{ Monthly pay } & $<1,500,000$ & 48 & $2.06 \pm 0.81$ & $5.42^{*}$ \\
\hline & & $1,510,000-2,000,000$ & 130 & $2.09 \pm 0.76$ & \\
\hline & & $2,010,000-2,500,000$ & 57 & $2.37 \pm 0.82$ & \\
\hline & & $2,510,000-3,000,000$ & 42 & $2.26 \pm 0.89$ & \\
\hline & & $<3,010,000$ & 20 & $2.90 \pm 0.79$ & \\
\hline & \multirow[t]{3}{*}{ Physical therapist career period } & $<5$ years old & 113 & $2.06 \pm 0.79$ & $9.37^{*}$ \\
\hline & & $6-9$ years old & 106 & $2.14 \pm 0.81$ & \\
\hline & & $<10$ years old & 78 & $2.55 \pm 0.80$ & \\
\hline & \multirow[t]{2}{*}{ Position } & Manager of physical therapy & 76 & $2.41 \pm 0.87$ & $2.28^{*}$ \\
\hline & & Physical therapy & 214 & $2.16 \pm 0.80$ & \\
\hline & \multirow[t]{3}{*}{ Working week } & $<40$ times & 66 & $2.24 \pm 0.75$ & 0.07 \\
\hline & & 41-49 times & 208 & $2.21 \pm 0.85$ & \\
\hline & & $<50$ times & 23 & $2.26 \pm 0.86$ & \\
\hline & \multirow[t]{2}{*}{ Site } & Gwangju & 226 & $2.19 \pm 0.80$ & -1.23 \\
\hline & & Jeonnam & 71 & $2.32 \pm 0.89$ & \\
\hline & Service period & $<5$ years old & 218 & $2.14 \pm 0.83$ & 4.07 \\
\hline & & $6-9$ years old & 58 & $2.45 \pm 0.78$ & \\
\hline & & $<10$ years old & 21 & $2.43 \pm 0.81$ & \\
\hline & Part & Neurological physical therapy & 65 & $2.22 \pm 0.80$ & 0.37 \\
\hline & & Electrotherapy physical therapy & 134 & $2.14 \pm 0.79$ & \\
\hline & & Pediatric physical therapy & 43 & $2.28 \pm 0.88$ & \\
\hline & & Orthopedic physical therapy & 55 & $2.36 \pm 0.89$ & \\
\hline & 1 day number of patient care & $<10$ peoples & 25 & $2.36 \pm 0.95$ & 1.12 \\
\hline & & $11-20$ peoples & 67 & $2.24 \pm 0.74$ & \\
\hline & & 21-30 peoples & 71 & $2.32 \pm 0.73$ & \\
\hline & & 31-40 peoples & 74 & $2.04 \pm 0.90$ & \\
\hline & & <41 peoples & 47 & $2.26 \pm 0.90$ & \\
\hline & & And so on & 13 & $2.15 \pm 0.69$ & \\
\hline Are you satisfied with the operation & Sex & Male & 97 & $2.86 \pm 0.69$ & 0.98 \\
\hline method of continuing education? & & Female & 200 & $2.77 \pm 0.71$ & \\
\hline & Age & 20-29 years old & 116 & $2.72 \pm 0.67$ & $3.57^{\star}$ \\
\hline & & 30-39 years old & 126 & $2.78 \pm 0.70$ & \\
\hline & & <40 years old & 55 & $3.01 \pm 0.76$ & \\
\hline & Education & College graduate & 177 & $2.76 \pm 0.76$ & $3.63^{*}$ \\
\hline & & University graduate & 97 & $2.78 \pm 0.70$ & \\
\hline & & A postgraduate school & 23 & $3.17 \pm 0.49$ & \\
\hline & Marital status & Unmarried & 183 & $2.75 \pm 0.05$ & -1.52 \\
\hline
\end{tabular}


Table 3. Continued

\begin{tabular}{|c|c|c|c|c|c|}
\hline \multirow{2}{*}{\multicolumn{2}{|c|}{ Characteristics }} & & $\mathrm{N}$ & $M \pm S D$ & tor $F$ \\
\hline & & Married & 114 & $2.88 \pm 0.07$ & \\
\hline & \multirow[t]{2}{*}{ Dependent family } & No & 171 & $2.77 \pm 0.69$ & -0.91 \\
\hline & & Yes & 126 & $2.84 \pm 0.73$ & \\
\hline & \multirow[t]{5}{*}{ Monthly pay } & $<1,500,000$ & 48 & $2.73 \pm 0.82$ & 1.12 \\
\hline & & $1,510,000-2,000,000$ & 130 & $2.75 \pm 0.65$ & \\
\hline & & $2,010,000-2,500,000$ & 57 & $2.79 \pm 0.75$ & \\
\hline & & $2,510,000-3,000,000$ & 42 & $2.90 \pm 0.69$ & \\
\hline & & $<3,010,000$ & 20 & $3.05 \pm 0.69$ & \\
\hline & \multirow[t]{3}{*}{ Physical therapist career period } & $<5$ years old & 113 & $2.73 \pm 0.69$ & $3.86^{*}$ \\
\hline & & $6-9$ years old & 106 & $2.74 \pm 0.76$ & \\
\hline & & $<10$ years old & 78 & $2.99 \pm 0.63$ & \\
\hline & \multirow[t]{2}{*}{ Position } & Manager of physical therapy & 76 & $2.88 \pm 0.67$ & 1.07 \\
\hline & & Physical therapy & 214 & $2.78 \pm 0.71$ & \\
\hline & \multirow{3}{*}{ Working week } & $<40$ times & 66 & $2.89 \pm 0.66$ & 1.03 \\
\hline & & 41-49 times & 208 & $2.76 \pm 0.74$ & \\
\hline & & $<50$ times & 23 & $2.87 \pm 0.46$ & \\
\hline & \multirow[t]{2}{*}{ Site } & Gwangju & 226 & $2.79 \pm 0.71$ & -0.26 \\
\hline & & Jeonnam & 71 & $2.82 \pm 0.68$ & \\
\hline & \multirow[t]{3}{*}{ Service period } & $<5$ years old & 218 & $2.74 \pm 0.69$ & 3.15 \\
\hline & & $6-9$ years old & 58 & $2.29 \pm 0.77$ & \\
\hline & & $<10$ years old & 21 & $3.05 \pm 0.74$ & \\
\hline & \multirow[t]{4}{*}{ Part } & Neurological physical therapy & 65 & $2.80 \pm 0.62$ & 0.99 \\
\hline & & Electrotherapy physical therapy & 134 & $2.80 \pm 0.69$ & \\
\hline & & Pediatric physical therapy & 43 & $2.81 \pm 0.79$ & \\
\hline & & Orthopedic physical therapy & 55 & $2.78 \pm 0.79$ & \\
\hline & \multirow[t]{6}{*}{1 day number of patient care } & $<10$ peoples & 25 & $2.80 \pm 0.87$ & 0.27 \\
\hline & & 11-20 peoples & 67 & $2.79 \pm 0.66$ & \\
\hline & & 21-30 peoples & 71 & $2.87 \pm 0.63$ & \\
\hline & & 31-40 peoples & 74 & $2.76 \pm 0.79$ & \\
\hline & & $<41$ peoples & 47 & $2.79 \pm 0.72$ & \\
\hline & & And so on & 13 & $2.69 \pm 0.48$ & \\
\hline
\end{tabular}

$* \mathrm{p}<0.05$

$\mathrm{M} \pm \mathrm{SD}$, mean \pm standard deviation.

while $67.3 \%$ answered "neutral" when asked "do you think the number of continuing education programs is reasonable?". Moreover, $55.9 \%$ of the responses were "neutral" when the question of "can your employer pay for your continuing education?" while $56.9 \%$ of the respondents answered "satisfied" when asked "do you think continuing education needs to be improved?". What is more, $37.7 \%$ of them answered "weekends or holidays" when asked "when do you think it is most suitable to participate in continuing education?". As for the question of "how many hours a day do you think are appropriate for continuing education?" $76.1 \%$ answered "8 hours a day", and with regard to the question of "how do you think continuing education should be delivered?" a majority of them $55.6 \%$ preferred the "lectures". To the question of "have you ever taken online courses as part of continuing education?", 90.2\% answered "no". In addition, a lion's share of the respondents $41.8 \%$ preferred "online education" when asked "how do you want to continuing education courses?" (Table 4).

\section{Measures to improve continuing education}

With respect to the improvement measures for continuing education, $54.9 \%$ of the respondents said that quantitative improvement was needed in educational content while $66.0 \%$ of them said that a reduction in the program fee was needed. A total of $73.1 \%$ answered "no" to a need for high-quality teaching staff while $54.9 \%$ said there was "no" need for a variety of educational programs. In addition, 92.3\% answered "no" to a need for more advertising for continuing education while $85.2 \%$ said that there was "no" need for improvement in educational environment. Moreover, $89.9 \%$ of the respondents answered "no" to a need for improvement in the attitude of participation among physical therapists while there was a large number of a response (62.0\%) of "no" for a need for special programs in specialized fields (Table 5).

\section{DISCUSSION}

The present study aims to provide baseline data to improve the 
Table 4. The need for continuing education

\begin{tabular}{|c|c|c|c|}
\hline Characteristics & & $\mathrm{N}$ & $\%$ \\
\hline Do you satisfied with the contents of the continuing education? & $\begin{array}{l}\text { Very dissatisfied } \\
\text { Dissatisfied } \\
\text { Neutral } \\
\text { Satisfied } \\
\text { Very satisfied }\end{array}$ & $\begin{array}{r}255 \\
9 \\
24 \\
3 \\
6\end{array}$ & $\begin{array}{r}85.9 \\
3.0 \\
8.1 \\
1.0 \\
2.0\end{array}$ \\
\hline Do you think continuing education is need? & $\begin{array}{l}\text { Very dissatisfied } \\
\text { Dissatisfied } \\
\text { Neutral } \\
\text { Satisfied } \\
\text { Very satisfied }\end{array}$ & $\begin{array}{r}27 \\
59 \\
145 \\
58 \\
8\end{array}$ & $\begin{array}{r}9.1 \\
19.9 \\
48.8 \\
19.5 \\
2.7\end{array}$ \\
\hline How helpful do you think are continuing education in your work? & $\begin{array}{l}\text { Very dissatisfied } \\
\text { Dissatisfied } \\
\text { Neutral } \\
\text { Satisfied } \\
\text { Very satisfied }\end{array}$ & $\begin{array}{r}72 \\
94 \\
120 \\
10 \\
1\end{array}$ & $\begin{array}{r}24.2 \\
31.6 \\
40.4 \\
3.4 \\
0.3\end{array}$ \\
\hline Do you think reasonable number of continuing education? & $\begin{array}{l}\text { Very dissatisfied } \\
\text { Dissatisfied } \\
\text { Neutral } \\
\text { Satisfied } \\
\text { Very satisfied }\end{array}$ & $\begin{array}{r}9 \\
66 \\
200 \\
16 \\
6\end{array}$ & $\begin{array}{r}3.0 \\
22.2 \\
67.3 \\
5.4 \\
2.0\end{array}$ \\
\hline Can you work place pay the fees for continuing education? & $\begin{array}{l}\text { Very dissatisfied } \\
\text { Dissatisfied } \\
\text { Neutral } \\
\text { Satisfied } \\
\text { Very satisfied }\end{array}$ & $\begin{array}{r}80 \\
48 \\
166 \\
3 \\
0\end{array}$ & $\begin{array}{l}26.9 \\
16.2 \\
55.9 \\
1.0 \\
0\end{array}$ \\
\hline Do you think continuing education need to be improved? & $\begin{array}{l}\text { Very dissatisfied } \\
\text { Dissatisfied } \\
\text { Neutral } \\
\text { Satisfied } \\
\text { Very satisfied }\end{array}$ & $\begin{array}{r}3 \\
12 \\
57 \\
169 \\
56\end{array}$ & $\begin{array}{r}1.0 \\
4.0 \\
19.2 \\
56.9 \\
18.9\end{array}$ \\
\hline When do you think is the most suitable to participate in continuing education? & $\begin{array}{l}\text { Weekday mornings } \\
\text { Weekday afternoons } \\
\text { Weekday evenings } \\
\text { Weekends or holidays } \\
\text { Any time } \\
\text { And so on }\end{array}$ & $\begin{array}{r}54 \\
50 \\
49 \\
112 \\
23 \\
9\end{array}$ & $\begin{array}{r}18.2 \\
16.8 \\
16.5 \\
37.7 \\
7.7 \\
3.0\end{array}$ \\
\hline How many hours a day do you think are appropriate for continuing education? & $\begin{array}{l}8 \text { hours a time } \\
4 \text { hours at a time two separate } \\
\text { Many times } \\
\text { Doesn't matter } \\
\text { And so on }\end{array}$ & $\begin{array}{r}226 \\
39 \\
13 \\
12 \\
7\end{array}$ & $\begin{array}{r}76.1 \\
13.1 \\
4.4 \\
4.0 \\
2.4\end{array}$ \\
\hline How do you think continuing education should be delivered? & $\begin{array}{l}\text { Lectures } \\
\text { Consultation and discussion } \\
\text { Presentation of case study } \\
\text { Practicum } \\
\text { Field trips } \\
\text { And so on }\end{array}$ & $\begin{array}{r}165 \\
4 \\
55 \\
43 \\
14 \\
16\end{array}$ & $\begin{array}{r}55.6 \\
1.3 \\
18.5 \\
14.5 \\
4.7 \\
5.4\end{array}$ \\
\hline Have you ever taken continuing education courses online? & $\begin{array}{l}\text { Yes } \\
\text { No }\end{array}$ & $\begin{array}{r}29 \\
268\end{array}$ & $\begin{array}{r}9.8 \\
90.2\end{array}$ \\
\hline How do you want to take continuing education courses? & $\begin{array}{l}\text { Offline education } \\
\text { Online education } \\
\text { Offline and online education }\end{array}$ & $\begin{array}{r}70 \\
124 \\
103\end{array}$ & $\begin{array}{l}23.6 \\
41.8 \\
34.7\end{array}$ \\
\hline
\end{tabular}

quality of continuing education by investigating the current status of continuing education programs for physical therapists and their perception of and needs for continuing education.

Since a majority of the participants in the continuing education were between 30 and 39 years old, unmarried and working for five years or less, it is thought that they might need continuous self-de- velopment with regard to physical therapy approaches. Therefore, the education content related to professional expertise or physical therapy should be taken as a priority. ${ }^{16}$ In addition, it appears that many of the respondents were working at clinics since about half of them were in charge of electrotherapy physical therapy. Hence, continuing education should also focus on the expert knowledge such 
Table 5. Measures to improve continuing education

\begin{tabular}{llrc}
\hline Characteristics & & N & $\%$ \\
\hline Qualitative improvement of educational content & No & 134 & 45.1 \\
& Yes & 163 & 54.9 \\
High-quality instructor staff & No & 217 & 73.1 \\
& Yes & 80 & 26.9 \\
A variety of educational programs & No & 163 & 54.9 \\
& Yes & 134 & 45.1 \\
Reduction in program fees & No & 101 & 34.0 \\
More advertising of continuing education & Yes & 196 & 66.0 \\
& No & 274 & 92.3 \\
Improvement of educational environment & Yes & 23 & 7.7 \\
& No & 253 & 85.2 \\
Attitude of participating physical therapist & Yes & 44 & 14.8 \\
& No & 267 & 89.9 \\
Provision of special lectures on specific fields & Yes & 30 & 10.1 \\
& No & 184 & 62.0 \\
& Yes & 113 & 38.0 \\
\hline
\end{tabular}

as how to treat chronic pain or aftereffects of a car accident which are often dealt with at clinics.

Particularly, most of the respondents were found to be dissatisfied with fees for continuing education and significant differences were largely observed in the general characteristics of a program fee. As such, the association of physical therapy should explore various ways for improvement, including group discounts as well as individual discounts for outstanding members and people who have taken continuing education for an extended period. In addition, it appears that such dissatisfaction stemmed from the involuntary participation of physical therapists in the current continuing education which is mandatory in the medical license reporting system. Therefore, for future satisfaction, there needs to be gradual improvement. ${ }^{17}$ Selecting the education program topics through a needs analysis among education consumers or through the cooperation with educational institutions is deemed to be an excellent idea. ${ }^{18,19}$

Many physical therapists see a need for continuing education and think that it is helpful to their work. Though they are somewhat satisfied or satisfied with the number and hours of education at large, most of them expressed strong dissatisfaction with having to participate in education. This is partly because most of the respondents have never taken online continuing education courses offered by the association of physical therapy, and it appeared that many of them preferred either online continuing education courses or a combination of online of offline continuing education courses.
This shows that many physical therapists would want more online education which is not bound by time and space, and the therapists who received continuing education in the past would want to complement the necessary knowledge and skills, and not have just a one-off education system. Moreover, while most of the respondents were in agreement to continue with the current eight hours of education while a few said they would rather divide them into two to four hours a day or more. At present, the number of hours of the continuing education for dental hygienists is eight hours which is the same as that of physical therapists but the education takes place both in the first and second halves of a year and the dates and contents of the education are advertised on the association's websit., ${ }^{914}$ In addition, with regard to the improvement measures for continuing education, many respondents answered that the quality of educational content should be improved along with a reduction in educational fees. Therefore, there is a need to diversify continuing education through online education and various other means and to change it with a diverse teaching staff. ${ }^{16,20}$ In addition, it is thought that there needs to be program development in continuing education and practical provision of continuing education while working on the job at hospitals and clinics.

According to the findings of the present study, the satisfaction level of physical therapists in Gwangju and Jeonnam with continuing education was found to be average while the quality improvement of continuing education and reduction in the program fee were shown to be needed most urgently. In addition, though the respondents have rarely taken continuing education online, since their preference for online education was the highest, there needs to be more efforts to promote the online continuing education programs.

\section{REFERENCES}

1. Shin HS, Kim JS. Factors affecting emergency room nurse job in small and medium sized hospitals. J Kor Nurs Adm Acad . 2015;21(4):386-92.

2. Lee IH, Shin AM, Son CS et al. Understanding the behavior of physical therapists and occupational therapists in protecting patient's medical information - an application of the theory of planned behavior. J Kor Phys Ther. 2010;22(2):55-60.

3. Lee MY, Chung JJ, Hong HJ et al. Evaluation of the home-based rehabilitation program based on CBR model through in-depth interviews with visiting physical therapists. J Kor Phys Ther. 2015;27(1):68-77.

4. Lee KH, Jung BO. A study on the relationship between professional self- 
concept and job satisfaction of physical therapists. J Kor Phys Ther. 2009; 21(3):109-18.

5. Kim MJ, Han SS. Comparison of job satisfaction, commitment to organization, nursing organization culture and job experience between national/public hospital nurses and private hospital nurses. J East-West Nurs. 2007;13(1):22-30.

6. Kim MH, Kim GE, Kim JH et al. Changes in awareness of major employment following clinical practice in students of physical therapy. J Kor Phys Ther. 2014;26(6):403-10.

7. Park JH, Oh HY et al. Health \& medical laws. Seoul, PanMun education, 2016:153-4.

8. Kim MJ, Shin GR, Yu JS et al. A survey on continuing education of nurse. Kor Nurs. 1996;35(5):55-64.

9. Moon JK, Song BK, Hwang BY. A study on job satisfaction among physical therapists in public health centers of the seoul metropolitan area. J Kor Phys Ther. 2010;22(2):61-8.

10. Jung JY. A study on the evaluation of management of continuing education programs for the improvement of the job performance of dental hygienists. Dankook University. Dissertation of Doctorate Degree. 2014.

11. Ahn SY. Development of assessment tool for continuing education of dental hygienists. Hanyang University. Dissertation of Doctorate Degree. 2013.

12. Lee MA. Analysis of the effects of job characteristics on their job satis- faction among physical therapists. Kyunghee University. Dissertation of Master's Degree. 2013.

13. Na JM. A study on medical practitioners' perceptions on continuing medical education. Dissertation of Master's Degree. 2012.

14. Park KH. The role of medical record technician and survey of continuing education needs. Yonsei University. Dissertation of Master's Degree. 2000.

15. Park JH. A consideration on the systems related to physical therapist license in korea. Dissertation of Master's Degree. 2006.

16. Kim JD. The present condition of continuing education for physical therapists and needs. Chonnam University. Dissertation of Master's Degree. 2016.

17. Lee YJ. The research on the reality and recognition relayed to inservice education of the nurse. Andong University. Dissertation of Master's Degree. 2005.

18. Lee YG. A study on the need for continuing education programs for nurses working in medium-sized hospitals. Yonsei University. Dissertation of Master's Degree. 2007.

19. Kwon MJ. A survey of evidence-based practice of physical therapist. J Kor Phys Ther. 2006;18(3):23-6.

20. Park SK, Heo JW. Correlation between professionalism, job satisfaction and job performance of the physical therapist. J Kor Phys Ther. 2015; 27(1):12-7. 